\section{TREATMENT OF RINGWORM OF THE SCALP}

BY

\section{H. HABER, M.D.Prague}

- R. T. BRAIN, M.D., F.R.C.P.

AND

J. W. HADGRAFT, Ph.C., A.R.I.C.

(From St. John's Hospital for Diseases of the Skin and the Royal Free Hospital)

The treatment of ringworm of the scalp by the application of preparations containing fungicides in various penetrating vehicles was the subject of an investigation by Brain et al. (1948). The best results were obtained with an ointment containing phenylmercuric nitrate $0.5 \%$ in a base consisting of "Crill No. 6," which is a non-ionizing surface-active compound, and "carbowax 1500 " buffered to $p \mathrm{H} \mathrm{4-5}$ by the addition of a solution of citric acid and sodium propionate. This investigation has now been extended to the use of salicylanilide in the same ointment base.

Salicylanilide is a white or pale-cream powder, very sparingly soluble in water, but soluble in alcohol and in the ointment base described above. Its use in the treatment of ringworm infections was first reported in America by Schwartz et al. (1946), who used an ointment containing salicylanilide $4.5 \%$, hyamine $1 \%$, and carbowax 1500 $94.5 \%$. Hyamine is a quaternary ammonium compound having surface-active properties, and was included to promote penetration of the fungicide into the hair follicle.

More recently the treatment of ringworm of the scalp with salicylanilide has been investigated in Scotland by Kinnear and Rogers (1948). These workers used an ointment containing salicylanilide $4.5 \%$, cetrimide (" cetavlon ") $1 \%$, and carbowax $150094.5 \%$. It is significant that they also used salicylanilide in a "eucerin" base, and found that it was no more effective than other ointments, whilst the conclusion was reached that salicylanilide in the carbowax base was the only preparation investigated which gave satisfactory results.

The following is the report on 27 cases of ringworm of the scalp treated with an ointment which has the following composition :

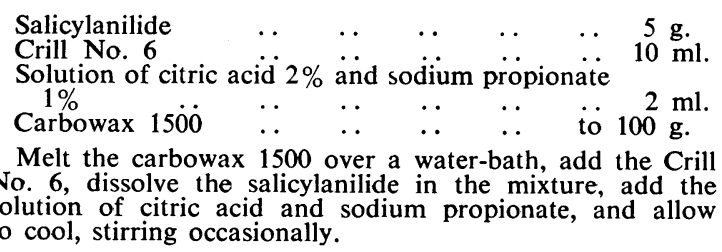

\section{Instructions to Parents}

The same technique of treatment was employed as in the previous cases. Parents were given written instructions and advised to follow them meticulously. The instructions were as follows :

1. Cut the hair of the entire scalp very short and keep it short throughout the treatment.

2. A linen cap should be worn day and night.

3. Wash the scalp every morning with soap and hot water and scrub the affected parts with a soft nailbrush.

4. Apply the ointment to the lesions with the help of a toothbrush and spread the rest of it into the entire scalp.

- Report to the hospital should any undue irritation occur.
Remarks on Instructions.-(1) It has been found that whenever the mother failed to cut the hair of the entire scalp infected patches or individual hairs escaped the effect of the treatment. This made a prolongation of treatment necessary. (2) The linen cap prevented the spread of ringworm from infected pillows and hats, and protected the rest of the children in the family from contracting the disease. Several examinations of the inner side of headgears under Wood's light revealed the presence of fluorescing stumps. (3) We still think that mechanical friction is of considerable assistance in the elimination of infected hairs, the epilation being the preliminary step to any effective treatment of ringworm. Our further observations (see below) confirm that. (4) The toothbrush is an additional mechanical factor, and is also a good applicator. (5) Severe reactions such as kerion were not sought, and when these occurred parents were told to report as soon as possible in order to control the inflammation.

Parents were given enough ointment to last two to three weeks, and throughout the treatment the patients were seen only three or four times.

\section{Clinical Picture}

Every single case was diagnosed clinically, confirmed by Wood's light and microscopy, and the fungus in question identified by culturing. Both the course of the treatment and the follow-up were controlled with the help of a watchmaker's glass. In the experience of one of us (H. H.) it has proved indispensable in assessing the real progress of each case. It was interesting to watch the behaviour of the infected patches from the first day of the examination to the time of discharge of the case.

Under a watchmaker's glass by daylight an infected patch shows characteristic features which make a clinical diagnosis possible: the infected hair is broken, it has lost its elasticity, and it can be laid in different directions without returning to its previous position. It appears slightly darker, owing to the loss of the hair cuticle.

When looked at under Wood's light with the help of a watchmaker's glass the infected hairs appear brilliantly green, like the fluorescent dial of a wrist-watch, and the hair stumps lie loosely on the scalp but are still fixed in their follicles. When treatment has started, and the case is seen after a period of several weeks, a slightly different picture is presented. The hair stumps are polished off and the infected hairs appear now as brightly shining dots like stars on a dark background, and, when the case is responding, rarefaction of the patch is demonstrable. This rarefaction is due to the fact that a few hairs have already started to fall out, so that when the patch has become negative to Wood's light it does not mean that the affected hair has lost its fluorescence, but that epilation has occurred. As a matter of fact, a cured case shows the patch well polished and smooth, whereas a case which has not responded remains covered with hair stumps, and this fact can always be established by examining the patch with bare fingers. The lesions which have responded are smooth to the touch, whereas those that have not responded feel like a fine grater.

After a few weeks the empty follicles become filled with parakeratotic scales, and when such a patch is examined under Wood's light it seems as if the follicles contain white fluorescent hair stumps, indicating a relapse; but when removed with the help of forceps, placed on a slide with a drop of liquor potassae, and examined under the microscope it proves to consist of dried epidermis cells. Sometimes a few real white hair stumps were removed and proved to contain dry dead spores within them. They were of no significance.

Whenever pustulation or kerion formation occurred (which was accidental), both mechanical and ointment 
treatments were stopped at once. A lotion containing acriflavine 1 in 1,000 with $10 \%$ tannic acid was applied until the lesions subsided. Crusting and scaling were treated with a sulphur-and-salicylic ointment. Individual infected hairs were dealt with by epilation with forceps.

The usual routine was to examine the patients every two, three, or four weeks. Once the case had become negative the mother was advised to continue with the treatment for another week, after which the treatment was stopped. A monthly follow-up for at least three months was necessary before the cases were discharged as cured, but we tried to extend surveillance as long as possible, and succeeded in a great number of cases in maintaining it up to five, six, or seven months.

\section{Analysis of Cases}

The accompanying Table gives a detailed account of the course of treatment of the 27 cases.

Cases of Microsporon felineum.-There were 20 cases in this group. Seven boys and 13 girls. The age ranged between 3 and 11 years. Fifteen cases were cured by salicylanilide alone. The time of treatment was between three and 14 weeks. Three cases, after having been treated with salicylanilide for ten weeks were given cảrbowax ointment containing phenylmercuric nitrate. They were cured in 7,8 , and 10 weeks. Two cases were epilated by $x$ rays. Out of 20 cases, 15 responded and five failed.

Cases of $M$. audouini.-Six cases, all boys between 4 and 8 years; four were cured-two in six weeks, one in 12. and one in 16 . Two cases failed, and were cured by $x$-ray epilation ( $75 \%$ cure, $25 \%$ failure).

Case of Trichophyton sulphureum.-A 6-year-old boy proved to be very resistant to local treatment, and after four months had to be referred to the radiotherapist for epilation.

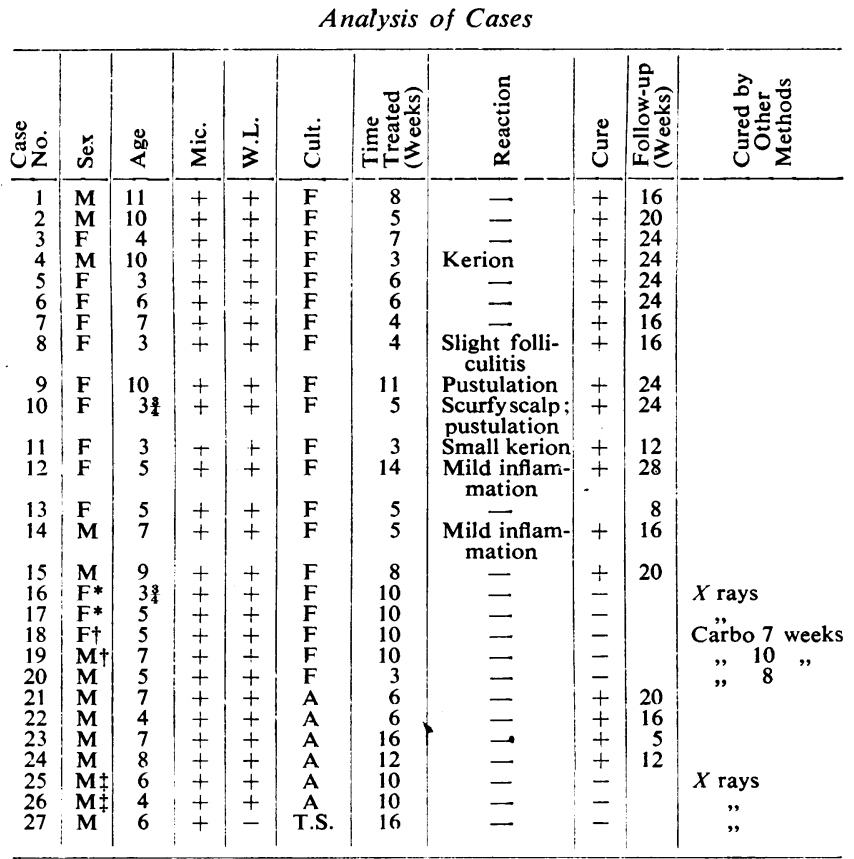

* Sisters. † Brother and sister. ¥ Brothers.

TS $=$ Tr sulphureum.

Carbo = Carbowax ointment containing $0.5 \%$ phenylmercuric nitrate.

\section{Comment}

There was local reaction in seven cases, ranging from mild folliculitis and pustulation to true kerion formation. All occurred in $M$. felineum cases, none in the $M$. audouini cases.
Comparison of the results achieved with phenylmercuric nitrate with those achieved with salicylanilide reveals very little difference. It is not the fungicide which effects the cure but the penetrating power of the bases employed. The latter bring about a softening of the hair and a loosening of the connexion between the hair and the hair papilla which effect epilation. Any fungicide will cure when the hair is removed. $X$-ray epilation has to be followed by the application of a fungicide.

We would like to point out that in no case have we seen healthy hairs fall out; the removable hairs were always proved to be infected.

In our previous paper we expressed the opinion that variance of response to treatment might be due to the individual resistance of the fungus to the fungicide. This may indeed be the case, but we would like to add another factor which may be important in influencing response to local treatment: that it is probably the hair itself which resists penetration of the base. From the table it will be seen that three pairs of brothers and sisters failed to respond to local treatment. In our opinion this may have been due to an intrinsic quality in the hair of the same family which was resistant to local forms of therapy.

\section{Summary}

A report is given on the treatment of ringworm of the scalp by the application of $5 \%$ salicylanilide in carbowax 1500 .

Chemical properties of salicylanilide and the literature on its use in the treatment of ringworm are reviewed.

Twenty-seven cases-20 of $M$. felineum infection, six due to $M$. audouini, and one due to $T r$. sulphureum-were treated. Cure was effected in $75 \%$ of the cases.

No significant differences were observed between the responses to treatment of the human and of the animal type of ringworm.

The results obtained with salicylanilide in carbowax 1500 are practically identical with those achieved with carbowax ointment containing $0.5 \%$ phenylmercuric nitrate, as employed in our previous series.

We wish to express our thanks to Imperial Chemical (Pharmaceuticals) Ltd. for the supply of salicylanilide used in these investigations, and to Mr. R. C. Syms, chief technician at St. John's Hospital, for his valuable assistance.

\section{REFERENCES}

Brain, R. T.. Crow, K., Haber, H., McKenny, C., and Hadgraft, J. W. (1948). British Medical Journal, 1, 723. Kinnear, J., and Rogers, J. (1948). Ibid., 2, 854.

Schwartz, L., Peck, S. M., Botvinick, I., Leibovitz, A. L., and Frasier, E. S. (1946). J. Amer. med. Ass., 132, 58.

A proposal concerning the formation of a Department of Public Health in the Ministry of the Interior in Western Germany with, ancillary to it, an advisory council and a medical research council to deal with the standardization of drugs, vaccines, and sera is receiving favourable consideration by the German authorities, according to the July report of the Control Commission for Germany (British Element). As a result of a meeting in Geneva on June 1, 1949, at which a British and a German representative from Nordrhein-Westfalen were present, the World Health Organization has set up an International anti-Venereal-Disease Commission of the Rhine. This commission, consisting of experts on venereal disease from France, Holland, Belgium, Switzerland, and Germany-from the countries bordering the Rhine-with representatives of the Occupation Authorities of the Western Zones, made a technical study tour of the main venereal-disease treatment centres in the Rhine River area. This was the first step towards the co-ordination of the anti-venereal-disease services in the five countries concerned. 